Brown-HET-1499

\title{
Exact Statistics of Chaotic Dynamical Systems
}

\author{
Zachary Guralnik * \\ Deptartment of Physics \\ Brown University \\ Providence, RI 02912
}

\begin{abstract}
We present an inverse method to construct large classes of chaotic invariant sets together with their exact statistics. The associated dynamical systems are characterized by a probability distribution and a two-form. While our emphasis is on classical systems, we briefly speculate about possible applications to quantum field theory, in the context of generalizations of stochastic quantization.
\end{abstract}

*zack@het.brown.edu 


\section{Introduction}

Due to the sensitive dependence of chaotic systems on initial conditions, their behavior over sufficiently long times is unpredictable in practice, even though the underlying dynamics is deterministic. Instead of attempting to accurately describe a phase space trajectory over a long duration, it makes more sense to try to calculate statistical properties of the system, such as equal time moments and the frequency with which the system coordinates lie within different regions of phase space.

Typically, statistical properties are calculated by direct numerical simulation. The evolution of the dynamical system is observed on a computer, starting with a particular initial condition, and the statistics are computed from averages over data points generated at regular time intervals. It is assumed that the result converges to the exact statistics fast compared to the the rate at which numerical errors are amplified by the chaotic nature of the system.

From an aesthetic point of view, direct numerical simulation is not an appealing way to compute statistics, since this approach is not formulated solely in terms of the variables one is actually interested in, namely the statistical quantities. There are equations which deal directly with the statistics, such as an equation due to Hopf [1, 2] which governs the generating functional of equal time moments. A better known equation, related to Hopf's equation by Fourier transformation, is the Fokker-Planck equation describing the time evolution of a probability distribution over phase space. In some special systems, such as the one described in [3], an analytic solution for the statistics exists, even for a very large number of degrees of freedom.

In this article, we will describe an inverse method to construct chaotic dynamical systems, starting with an invariant probability distribution and a two-form having a relatively simple analytic structure. This method allows one to generate, in principle, infinite classes of dynamical systems for which some statistical properties are known exactly. While we give examples with three and four degrees of freedom, we do not anticipate prohibitive obstructions in applying the inverse method to systems with a very large number of degrees of freedom.

Our starting point is an analytical expression for an invariant probability distribution $\rho(\vec{x})$ which is, by construction, a smooth measure in an $N$ dimensional phase space. It well known that the geometry of a chaotic invariant set may be fractal, having a fractional information dimension less than $N$. However, even with information dimension less than $N$, an invariant distribution with support in $N$ dimensions may still be relevant to the statistics of a chaotic trajectory as a probability distribution on the chaotic invariant set upon projection to lower integer dimension. We give several examples where $\rho(\vec{x})$ describes the statistics of chaotic trajectories upon projection to lower dimension.

There are examples for which we have found numerical evidence that the initial distribution $\rho(\vec{x})$, with N-dimensional support, gives accurate results even for connected moments (cumulants) which do not admit projection to lower integer dimension, such as $\langle x y z\rangle_{c}$ in $N=3$. This may be because the information dimension, which we have not yet calculated, is very nearly $N$. However, we speculate that the Hopf functional $\Psi(\vec{j})=<\exp (i \vec{j} \cdot \vec{x})>$ may be sufficiently well behaved that its Fourier transform, which is by construction an invariant distribution, has support in $N$ dimensions and is equal to $\rho(\vec{x})$. If so, $\rho(\vec{x})$ determines all polynomial moments $\left\langle x^{n} y^{m} z^{l} \cdots\right\rangle$, although there should be other expectation values of functions on phase space which can not be obtained from $\rho(\vec{x})$, due to the fractional information dimension. We also find that, in many instances, the ratio of frequencies with which different regions of phase space are visited by a chaotic trajectory is determined by the distribution $\rho(\vec{x})$, even when the detailed geometry of the chaotic invariant set is quite complicated or unknown. 
Although our analysis is largely analytic, we have found that it is necessary to do some direct numerical simulations to determine if the initial invariant probability distribution $\rho$ (or rather its projection to lower dimension) is ergodic. For some choices of the initial distribution and two-form, the dynamics is not chaotic at all, in which case $\rho$ has the interpretation as an invariant distribution over periodic or quasi-periodic trajectories. In many other instances, chaotic trajectories do not visit all regions where $\rho$ has support, although the initial distribution can be rendered ergodic by restricting the domain of support and adjusting the normalization.

When an analytic expression can be given for the restricted domain of support, which can never be done with absolute certainty since these domains are determined numerically, we can make very well motivated conjectures for exact equal time moments. In the absence of an analytic expression for the restricted domain, one can not make statements about exact moments. However, we expect that $\rho$ still determines the ratios of frequencies with which a chaotic trajectory visits regions of phase space within which the chaotic invariant set has equivalent local geometry.

In the present work, we will focus on dynamical systems on $\mathbb{R}^{N}$, with polynomial velocity fields. However the inverse method we describe may be generalizable to dynamical systems in spaces with other topologies, such as classical spin systems on $S^{N} \otimes \mathbb{R}^{N}$.

The motivation for this work is several-fold. One intention is to provide exact solutions which can be used as testing grounds for theoretical ideas, and as benchmarks for approximate methods to compute the statistics of non-linear dynamical systems. Another intention is to provide a means to reverse engineer systems very close to ones of physical interest, although we will make no attempt to do so in this initial study. The principle difficulty in constructing physical systems seems to be the choice of two-form, which has no direct physical interpretation.

The organization of this article is as follows. In section 2 we briefly review the Fokker-Planck and Hopf equations governing the statistics of stochastic and chaotic dynamical systems. We introduce the inverse method in section 3 , and give examples of chaotic systems with three degrees of freedom and invariant probability distributions which are polynomial within their domain of support. In section 4, we give an example with four degrees of freedom. The inverse method for distributions with more complicated analytic structure is discussed in section 5 , where we give an example with 3 degrees of freedom. Conditions for uniqueness of the invariant distribution in the context of the inverse method are discussed in section 6 . In section 7 , we describe the inverse method for dynamical systems with Gaussian noise and a class of distributions which arise in the context of quantum field theory. Section 8 contains concluding remarks.

\section{Review of Hopf and Fokker-Planck equations}

Consider an $N$ dimensional dynamical system of the form

$$
\frac{d}{d t} x_{n}(t)=v_{n}[\vec{x}]+f_{n}(t)
$$

where $f_{n}(t)$ is a Gaussian random force for which

$$
\left[f_{n}(t)\right]=0, \quad\left[f_{m}(t) f_{n}\left(t^{\prime}\right)\right]=2 \Gamma \delta_{m n} \delta\left(t-t^{\prime}\right),
$$


and the brackets $[\cdots]$ indicate an average over the random variable. The time evolution of a probability distribution over phase space, $\rho(\vec{x}, t)$, satisfies the Fokker-Planck equation [4],

$$
\frac{\partial}{\partial t} \rho(t, \vec{x})=-\vec{\nabla} \cdot(\rho(t, \vec{x}) \vec{v}(\vec{x}))+\Gamma \vec{\nabla}^{2}(\rho(t, \vec{x})) .
$$

The Fourier transform of the probability distribution with respect to the phase space coordinates $\vec{x}$ is the Hopf characteristic functional [1, 2, 5]

$$
\Psi(\vec{j}, t)=<e^{i \vec{j} \cdot \vec{x}(t)}>,
$$

which generates equal time moments:

$$
\left.\left(-i \frac{\partial}{\partial j_{m}}\right)\left(-i \frac{\partial}{\partial j_{n}}\right) \cdots \Psi(\vec{j}, t)\right|_{j=0}=<x_{m}(t) x_{n}(t) \cdots>
$$

while $\ln (\Psi)$ generates the connected moments (or cumulants). The characteristic functional satisfies the equation

$$
-i \frac{\partial}{\partial t} \Psi=\hat{H} \Psi
$$

where $\hat{H}$, the Hopf Hamiltonian, is given by

$$
\hat{H} \equiv \sum_{m=1}^{N} \vec{j} \cdot \vec{v}\left(-i \frac{\partial}{\partial j}\right)-i \Gamma|\vec{j}|^{2} .
$$

In (2.7), the coordinates $x_{n}$ in the velocity function $v_{m}(\vec{x})$ have been replaced with the operators $-i \frac{\partial}{\partial j_{n}}$. The boundary conditions satisfied by $\Psi$ follow from the requirement that $\rho$ is a probability distribution. For instance

$$
\Psi(\vec{j}=0)=1, \quad \lim _{|\vec{j}| \rightarrow \infty} \Psi=0,
$$

with additional more complicated constraints arising from the positivity of $\rho$.

An invariant measure on phase space is one for which the statistics is static,

$$
\frac{\partial}{\partial t} \rho=0, \text { or } \frac{\partial}{\partial t} \Psi=0 .
$$

Invariant measures are naturally generated by trajectories of stochastic and deterministic chaotic dynamical systems with no explicit time dependence. The state of a chaotic system observed at sufficiently long time intervals looks random. Moreover the fraction of the time with which the system lies in a given region of phase space is assumed to converge as the observation time goes to infinity. This yields a distribution which is independent of when one starts to observe the chaotic system. Such a distribution is invariant (static) when viewed alternatively as a distribution over initial conditions. Note that the converse statement that an invariant measure describes the statistics of a chaotic trajectory is not always true. If it is true, the measure is said to be ergodic. Ergodic invariant measures have no convex decomposition, meaning that $\rho$ can not be written as $(1-x) \rho_{1}+x \rho_{2}$ where $\rho_{1,2}$ are independent invariant measures and $0<x<1$.

In some cases, such as the toy model of inviscid flow described in [6, 7], a zero mode of the Hopf Hamiltonian, satisfying $\hat{H} \Psi=0$, can be found exactly [3]. The zero mode corresponds to 
an ensemble with static statistics or, assuming ergodicity, to the statistics of a chaotic system observed over a sufficiently long period of time 1 . The work we discuss here amounts to an inverse approach of picking a zero mode and finding the Hamiltonian 2 . We will work with the Fokker-Planck equation, picking a probability density $\rho$ and finding a corresponding dynamical system, $\vec{v}(\vec{x})$. For the most part we will consider the deterministic case, although the application of the inverse method to a class of stochastic dynamical systems is discussed in section 7 .

\section{The inverse method for polynomial distributions}

Consider a deterministic dynamical system in an $N$ dimensional phase space,

$$
\frac{d}{d t} \vec{x}=\vec{v}(\vec{x})
$$

It is convenient to use the language of differential forms and define the velocity one-form $v=$ $v_{n} d x^{n}$, in terms of which the static, zero diffusion limit of the Fokker-Planck equation can be written as

$$
d^{*}(\rho v)=0
$$

One can therefore always (locally) write ${ }^{*}(\rho v)=d \mathcal{A}$ where $\mathcal{A}$ is an $N-2$ form 3 , or equivalently

$$
v=\frac{* d \mathcal{A}}{\rho} .
$$

Given any distribution $\rho$, we can find dynamical systems (characterized by $v$ ) such that $\rho$ is an invariant distribution by making a choice of $\mathcal{A}$. Since the Hodge dual of $\mathcal{A}$ is a two-form, it is more convenient at large $N$ to define the dynamics in terms of the probability distribution and the two-form ${ }^{*} \mathcal{A}$. A generic choice of $\rho$ and $\mathcal{A}$ will give a velocity $v$ which is not a polynomial function of the coordinates. In this article we will only consider polynomial $v$ on the phase space $\mathbb{R}^{N}$. Of course non-polynomial $v$ may also arise in various physical settings and for phase spaces with non-trivial topologies, such as classical spin systems.

In order to insure that $v$ is polynomial, let us define another $N-2$ form $\Omega \equiv \mathcal{A} / \rho^{2}$. Then

$$
{ }^{*} v=\rho d \Omega+2 d \rho \wedge \Omega
$$

in regions where $\rho \neq 0$. The simplest way to insure that $v$ is polynomial is to take polynomial $\rho$ and $\Omega$. However a polynomial $\rho$ is not normalizable, unless we restrict its range of support.

Let us therefore consider the following example for $N=3$.

$$
\begin{aligned}
& \rho=1-x^{4}-y^{2}-z^{6} \text { for } x^{4}+y^{2}+z^{6}<1, \\
& \rho=0 \text { for } x^{4}+y^{2}+z^{6} \geq 1,
\end{aligned}
$$

\footnotetext{
${ }^{1}$ The probability distribution related by Fourier transformation to the characteristic functional $\Psi$ found in 3] does seem to be ergodic.

${ }^{2}$ This kind of approach has been very useful in condensed matter physics, in which case one searches for the quantum mechanical Hamiltonian associated with a chosen ground state.

${ }^{3}$ For readers not familiar with differential forms, one can write $\rho \vec{v}=\vec{\nabla} \times \overrightarrow{\mathcal{A}}$ for the case $\mathrm{N}=3$. The differential form language proves useful for $N>3$.
} 
up to a normalization factor. To specify a dynamical system consistent with this invariant distribution, we must also make a choice of one form $\Omega$. Consider

$$
\Omega=(x y z) d y+\left(y^{2}\right) d z .
$$

With this choice, (3.13) gives the dynamical system:

$$
\begin{aligned}
& v_{x}=13 z^{6} x y-6 y^{3}-x y+2 y+y x^{5}-2 y x^{4}-2 y z^{6}+y^{3} x, \\
& v_{y}=8 x^{3} y^{2} \\
& v_{z}=-9 x^{4} y z+y z-y z^{7}-y^{3} z .
\end{aligned}
$$

Note that the equations (3.16) clearly do not permit passage across $y=0$ or $z=0$. Thus the initial distribution, which has support for positive and negative $y$ and $z$, can not be ergodic. Let us therefore consider a modified probability density

$$
\begin{aligned}
& \rho=1-x^{4}-y^{2}-z^{6} \text { for } y>0, z>0,1-x^{4}-y^{2}-z^{6}>0 \\
& \rho=0 \text { elsewhere, }
\end{aligned}
$$

up to normalization. Although this modification introduces delta functions in $\partial_{x} \rho$ and $\partial_{y} \rho$ at the $x=0$ and $y=0$ boundaries respectively, the Fokker Planck equation is still satisfied because $v_{x}(x=0)=0$ and $v_{y}(y=0)=0$. Figure 1 shows planar projections of points generated both by numerical simulation of this dynamical system, starting from the initial condition $(x, y, z)=$ $(0.5,0.5,0.5)$, and by random sampling according to the probability distribution (3.17). At the crude level of visual inspection, these seem to agree.
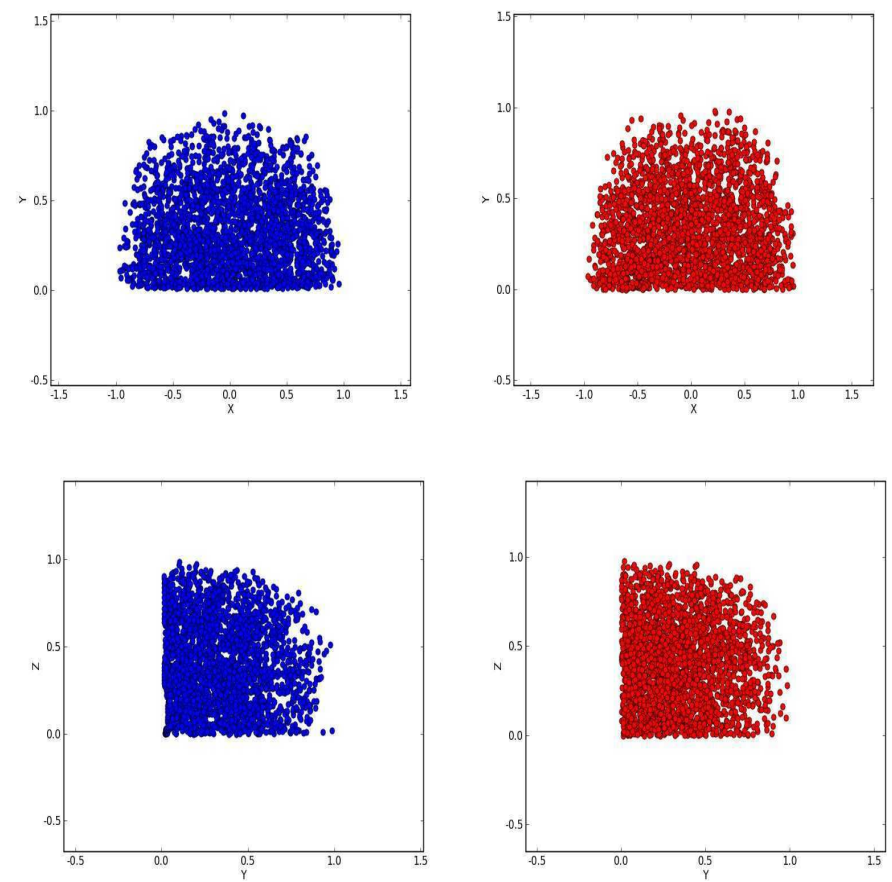

Figure 1. The XY and YZ projection of points generated by the dynamical system (3.16) observed at fixed time intervals $\Delta t=4$ (on the left) starting from the initial condition $(x, y, z)=$ $(0.5,0.5,0.5)$ and by random sampling with the probability distribution (3.17) (on the right). 
Numerical simulation over increasingly long time suggests that that the frequency with which the system lies in boxes in projections of the phase space converges to the box integral of the modified probability density. This last fact is a test of ergodic chaotic behavior and need not be generally true. If the system were not chaotic, the points generated by numerical simulation of the dynamics would not bear any resemblance to the points generated randomly according to the probability distribution. The probability distribution would then only have the interpretation as a distribution over initial conditions which is invariant under the evolution, but would have nothing to say about individual trajectories.

Direct numerical simulation also suggests that the equal time moments of the chaotic trajectory

$$
<x^{n} y^{m} z^{l}>\equiv \lim _{T \rightarrow \infty} \frac{1}{T} \int_{0}^{T} d t x(t)^{n} y(t)^{m} z(t)^{l}
$$

converge to those derived from the probability density (3.17),

$$
<x^{n} y^{m} z^{l}>=\int d^{3} x \rho(x, y, z) x^{n} y^{m} z^{l} .
$$

Moments calculated by direct numerical simulation of the dynamical system (3.16), and by Monte Carlo simulation using (3.17) are shown in table 1.

\begin{tabular}{c|c|c} 
Moments & Dynamics & Monte Carlo \\
\hline$<y>$ & 0.3449 & 0.3451 \\
\hline$<z>$ & 0.4223 & 0.4136 \\
\hline$<x^{2}>$ & 0.2066 & 0.2068 \\
\hline$<y^{2}>$ & 0.1720 & 0.1717 \\
\hline$<z^{2}>$ & 0.2387 & 0.2324 \\
\hline$<y z>$ & 0.1403 & 0.1386 \\
\hline$<y^{3}>$ & 0.1014 & 0.1011 \\
\hline$<z^{3}>$ & 0.1531 & 0.1493 \\
\hline$<x^{2} y>$ & 0.06562 & 0.06543 \\
\hline$<y^{2} z>$ & 0.06836 & 0.06721 \\
\hline$<y z^{2}>$ & 0.07670 & 0.07571 \\
\hline$<x^{4}>$ & 0.08531 & 0.08579 \\
\hline$<x^{2} y z>$ & 0.02607 & 0.02564 \\
\hline$<x^{2} y^{2}>$ & 0.03056 & 0.03021 \\
\hline$<x^{6} y^{4} z^{2}>$ & 0.0002604 & 0.0002482 \\
\hline$\vdots$ & $\vdots$ & $\vdots$
\end{tabular}

Table 1. The entries in the column labeled 'dynamics' were obtained by numerical simulation of the dynamical system (3.16) over a time duration 100000 , sampling at intervals $\Delta t=0.01$, starting from the initial conditions $(x, y, z)=(0.5,0.5,0.5)$. The column labeled 'Monte Carlo' was obtained by a hit and miss Monte Carlo calculation using the proposed exact distribution with 400000 accepted data points. 
As we have seen, the initial probability density which is used to generate the dynamical system may only describe a chaotic invariant set in a restricted domain. Although the restricted domain is simple here $\left(y>0, z>0, x^{4}+y^{2}+z^{6} \leq 1\right)$, it can also be much more complicated as we will see later. Even if the ratio of frequencies with which different regions of the invariant set are visited by the chaotic trajectory is determined by $\rho$, one must also know the geometry of the invariant set to calculate equal time moments (or the Hopf functional).

The present chaotic system is not dissipative. Trajectories with initial conditions outside the range of support of the initial probability distribution (3.14) avoid this region, or travel around the periphery and then move away. Thus we have reverse engineered a chaotic repeller starting from its statistics. Strictly speaking, since we can give numerical evidence for ergodicity of the distribution (3.17) (after projection), but not prove it, we have a very well motivated conjecture for the exact statistics of a chaotic trajectory.

At present, all the examples we have found of polynomial $\rho$ describing chaotic trajectories have real zeros which form a compact manifold $\Sigma$. Such systems are generally non-dissipative. For $\rho=0$ (3.13) becomes ${ }^{*} v=2 d \rho \wedge \Omega$, implying that the velocity is tangent to $\Sigma$. Thus trajectories outside the region bounded by the zeros of $\rho$ set can not enter the region inside. Construction of chaotic attractors by the inverse method requires that $\rho$ and the two-form ${ }^{*} \Omega$ have a more complicated analytic structure, examples of which are discussed in section 5.

The inverse approach allows one, to a limited extent, to reverse engineer chaotic invariant sets with a geometry of ones choosing. To illustrate some of the power and limitations of the approach, consider a polynomial probability distribution with support between spherical shells;

$$
\begin{aligned}
\rho & =\rho_{1} \rho_{2}, \\
\rho_{1} & =\left(3-x^{2}-y^{2}-z^{2}\right), \\
\rho_{2} & =\left(x^{2}+y^{2}+z^{2}-1\right),
\end{aligned}
$$

in the region where (3.21) and (3.22) are positive, and $\rho=0$ elsewhere. Together with a one form

$$
\Omega=(x+y) d y+z d x
$$

equation (3.13) yields the dynamical system

$$
\begin{aligned}
v_{x} & =8 z\left(x^{2}+y^{2}+z^{2}-2\right)(x+y), \\
v_{y} & =-10 z^{2} x^{2}-10 z^{2} y^{2}-9 z^{4}+20 z^{2}+4 x^{2}+4 y^{2}-3-x^{4}-2 x^{2} y^{2}-y^{4}, \\
v_{z} & =8 z y x^{2}+8 z y^{3}+8 y z^{3}-16 z y-9 x^{4}-8 x^{3} y-10 x^{2} y^{2}-8 x y^{3}-10 x^{2} z^{2} \\
& -8 x z^{2} y+20 x^{2}+16 x y+4 y^{2}+4 z^{2}-3-y^{4}-2 y^{2} z^{2}-z^{4} .
\end{aligned}
$$

Constant $z$ sections of a chaotic trajectory (figure 2) reveal considerably more structure than is present in the initial invariant distribution $\rho$ defined in (3.20)-(3.22). The chaotic invariant set fills regions between the concentric shells defined by $\rho$, although there are excluded regions within the support of $\rho$ which are not visited by the chaotic trajectory. We do not have analytic expressions for the excluded regions.

The initial invariant distribution used to generate dynamical systems by the inverse method seems to be related to the ergodic distributions associated with chaotic trajectories by a restriction of the domain of support. The restricted domain can very complicated, or even fractal. Without specifying properties of this domain, or its projections, one can at best give partial 
information about the exact statistics, such as the ratio of frequencies with which the chaotic trajectory visits different regions of phase space in which the chaotic invariant set has similar local geometry. To the limited numerical accuracy that we have checked for the present example, the frequency ratios are consistent with (3.20)-(3.22). However, we do not have a conjecture for equal time moments or the Hopf characteristic functional of this system.

Note that variations of the 2 form ${ }^{*} \Omega$ with fixed $\rho$ change the dynamics and the geometry of the chaotic invariant set (assuming the system remains chaotic). As an example, consider the distribution (3.20) with

$$
\Omega=x y z d x+x z d y+y d z .
$$

This gives a different dynamical system (we will not write down the rather long expression for the velocity field), with constant $\mathrm{z}$ sections of the chaotic trajectory plotted in figure 3 . We expect that the ratio of the visitation frequencies of different regions in the chaotic invariant set with equivalent local geometry are still given by $\rho$ in (3.20)-(3.22), but the equal time moments and the Hopf functional are different.

The statistics, in the form of the distribution $\rho$, may exhibit symmetries which are not respected by the dynamics. The distribution in (3.20), has rotational $S O(3)$ invariance, which is not respected by $\Omega$ in either (3.23) or (3.25), or in the geometry of the chaotic invariant set. The symmetry might be observed in ratios of frequencies with which different regions of the chaotic invariant set are visited, if not also in the moments or the Hopf functional. Such accidental statistical symmetries are very common. Note that the exact Hopf characteristic functional given for the Orszag-Mclaughlin model in [3] shows a rotational $S O(N)$ symmetry which the equations of motion do not exhibit.
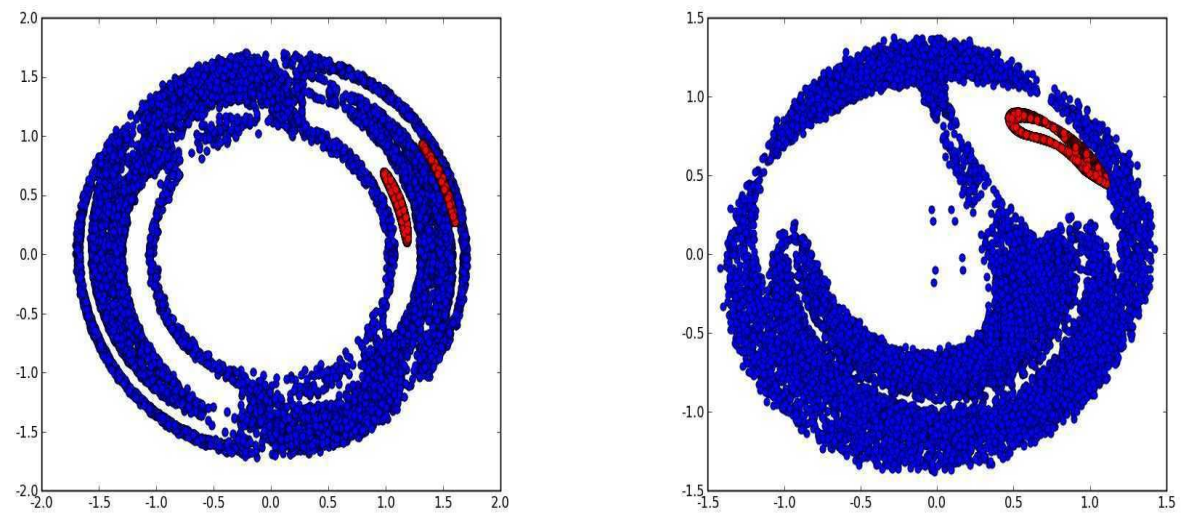

Figure 2. $z=0$ and $z=1.1$ sections of trajectories of the dynamical system defined by the invariant distribution (3.20) and the 1-form (3.23) for two sets of initial conditions, one of which belongs to the chaotic invariant set (dense blue dots) and the other which belongs to a quasiperiodic trajectory (loops of red dots). This is not a Poincaré section, since trajectories crossing the section in both directions have been plotted. The quasi-periodic orbit fills a two-torus in the full three dimensional phase space. 

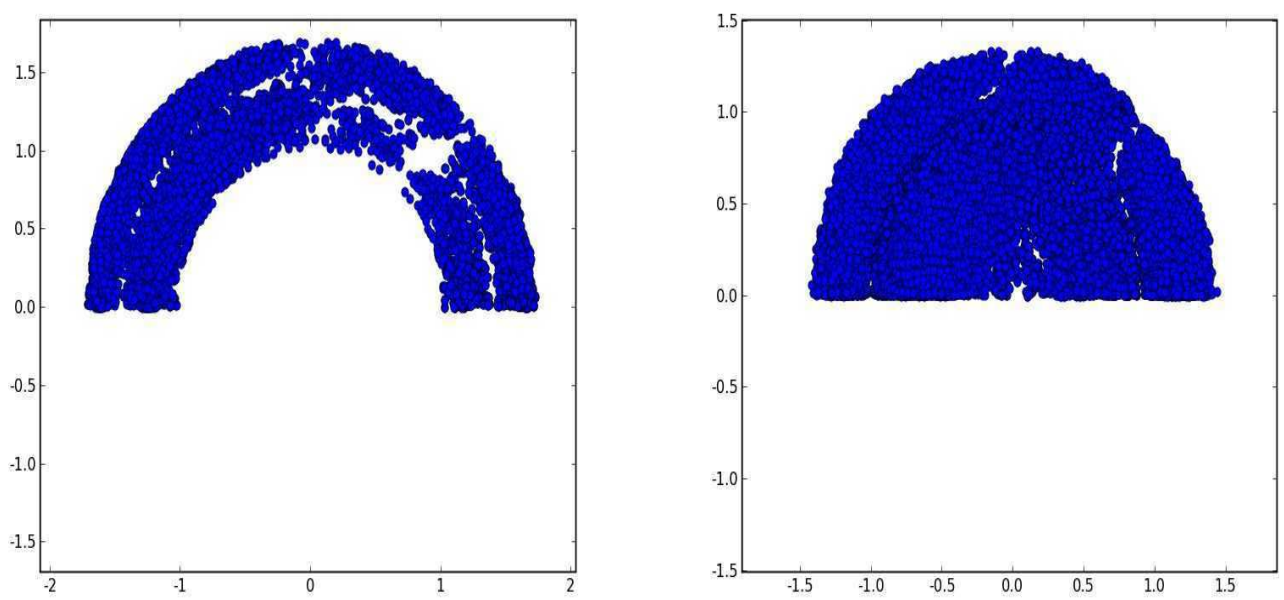

Figure 3. $z=0.1$ and $z=1.1$ sections of a chaotic trajectory associated with the dynamical system defined by the invariant distribution (3.20) and the 1-form (3.25).

\section{A four dimensional example}

Consider the invariant polynomial probability distribution

$$
\begin{aligned}
\rho & =\rho_{1} \rho_{2} \rho_{3} \\
\rho_{1} & \left.=1-\left(x_{1}^{2}+y_{1}^{2}\right)\right), \\
\rho_{2} & \left.=1-\left(x_{2}^{2}+y_{2}^{2}\right)\right), \\
\rho_{3} & =1-\left(x_{1}-x_{2}\right)^{2}-\left(y_{1}-y_{2}\right)^{2},
\end{aligned}
$$

up to a normalization factor, in the region where (4.27)-(4.29) are all positive, with $\rho=0$ elsewhere. To uniquely specify a corresponding dynamical system, we choose a two-form

$$
\Omega=x_{1} y_{1} d x_{2} \wedge d y_{2}+x_{2} y_{2} d x_{1} \wedge d y_{1}+x_{1}^{3} d x_{1} \wedge d y_{1}+x_{2}^{3} d x_{2} \wedge d y_{2} .
$$

Our choice of two-form is essentially random and not motivated by any physical constraint, besides the requirement that the system exhibit chaos with initial conditions inside the domain of support of $\rho$. Note that the simpler choice of two-form, $\Omega=x_{1} y_{1} d x_{2} \wedge d y_{2}+x_{2} y_{2} d x_{1} \wedge d y_{1}$ does not lead to chaotic dynamics. Applying (3.13) yields a velocity field with a large number of terms which we will not write explicitly 4 . Numerically simulating the resulting ODE for an initial condition within the domain of suport of $\rho$ suggests that the distribution $\rho$ is in fact ergodic upon projection to lower dimension. Figure 4 shows the $\left(x_{1}, y_{1}\right)$ and $\left(x_{1}, x_{2}\right)$ planar projections of points generated both by running the dynamical system and by random sampling according to the probability distribution (4.26). At the crude level of visual inspection, this distribution is consistent with the dynamics.

\footnotetext{
${ }^{4}$ This system has some vague resemblance to a classical spin system. However we are not (at this stage) attempting to reverse engineer either a physically motivated system or a simple system with a small number of terms. The operations on differential forms in (3.13) were all performed on a computer using code written in Python.
} 
Numerical simulation also suggests that the moments associated with the chaotic trajectory are consistent with those derived from the distribution $\rho$ in (4.26). Very good agreement is found for moments of the form $\left\langle x_{i}^{n} y_{i}^{m}\right\rangle$, even at high orders. Comparing the high order connected moments between degrees of freedom with both subscripts 1 and 2 seems to require longer run times than we have done at present. Numerical results for a few moments are recorded in table 2 .
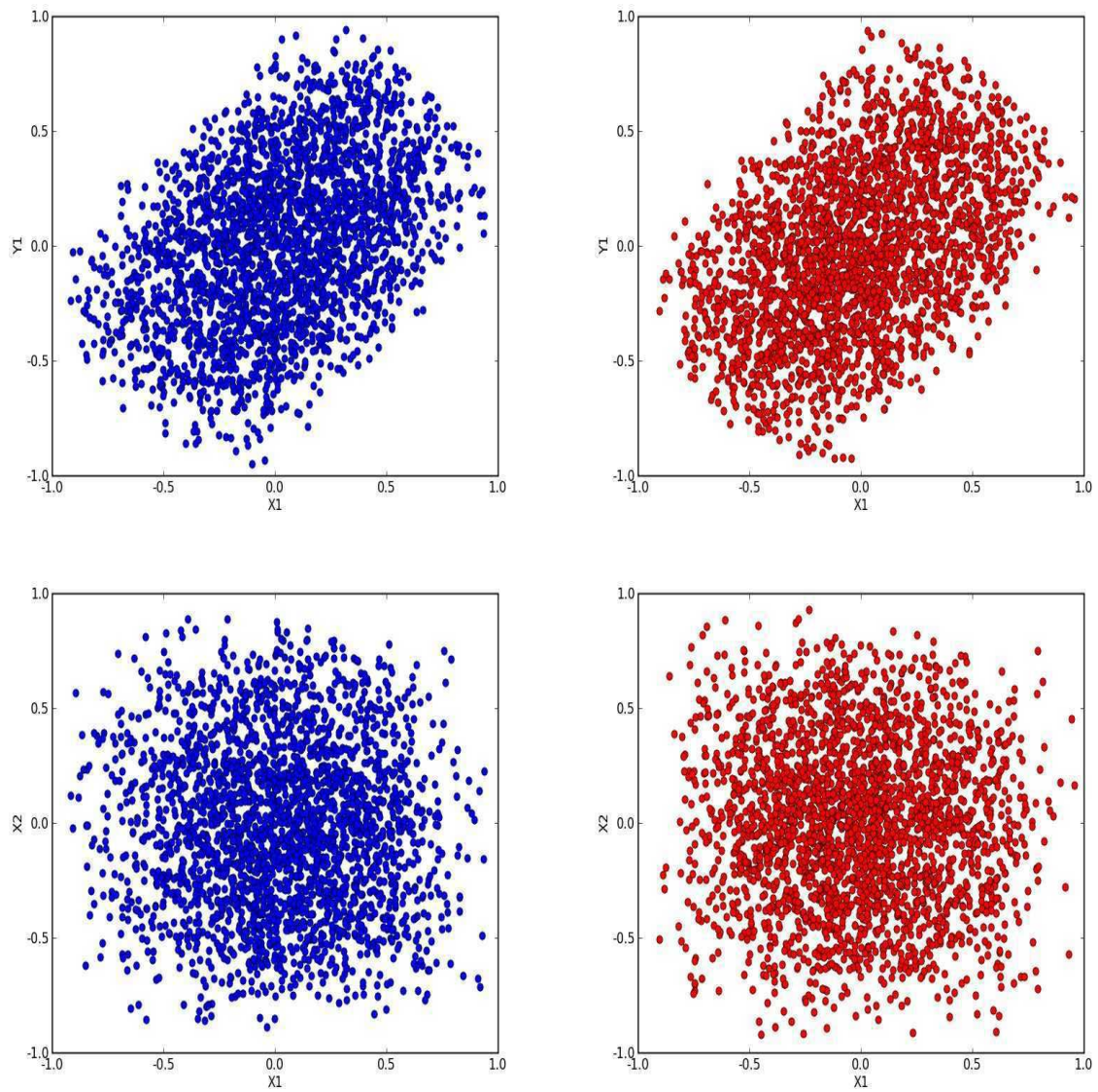

Figure 4. Projections to the $\left(x_{1}, y_{1}\right)$ and $\left(x_{1}, x_{2}\right)$ planes of points in phase space space generated by the chaotic trajectory observed at intervals $\Delta t=4$ (on the left) and randomly according the probability distribution (4.26) (on the right). 


\begin{tabular}{c|c|c} 
Moments & Dynamics & Monte Carlo \\
\hline \hline$<x_{1}^{2}>$ & 0.1285 & 0.1290 \\
\hline$\left.\left(<x_{1}^{2} y_{1}^{2}>-<x_{1}^{4}>-3<x_{1}^{2}>^{2}\right) /<x_{1}^{2}>^{2}>\right) /<x_{1}^{2}><y_{1}^{2}>$ & -0.6326 & -0.6098 \\
\hline$<x_{1}^{6} y_{1}^{6}>$ & -0.05425 & -0.05788 \\
\hline$<x_{1}^{12} y_{1}^{8}>$ & 0.0001276 & 0.0001287 \\
\hline$<x_{1}^{2} x_{2}^{2}>-<x_{1}^{2}><x_{2}^{2}>$ & $3.283 \times 10^{-6}$ & $3.277 \times 10^{-6}$ \\
\hline$<y_{1}^{2} y_{2}^{2}>-<y_{1}^{2}><y_{2}^{2}>$ & -0.0001122 & -0.0001387 \\
\hline$\vdots$ & -0.0001884 & -0.0001958 \\
\hline & $\vdots$ & $\vdots$
\end{tabular}

Table 2. The entries in the column labeled 'dynamics' were obtained by numerical simulation of the dynamical system. With the exception of the last two rows, the time duration was 20000, with data sampled at intervals $\Delta t=2$, starting from the initial conditions $\left(x_{1}, y_{1}, x_{2}, y_{2}\right)=$ $(0.1,0.2,0.11,0.09)$. The last two rows were obtained by sampling at intervals $\Delta t=4$ over a duration 400000. The column labeled 'Monte Carlo' was obtained by hit and miss Monte Carlo computation using the proposed exact distribution with 100000 accepted data points, for all but the last two rows. The last two rows were obtained with 400000 accepted data points.

\section{Non-polynomial distributions}

In the previous section we considered invariant distributions which were polynomial, with real zeroes forming a compact manifold. The inverse method may be readily generalized to more complicated analytic structures.

For example, consider distributions of the form,

$$
\rho=P e^{-Q},
$$

where $P$ and $Q$ are polynomia 5 . To insure that $v$ is polynomial, we now choose an $N-2$ form with the structure

$$
\Omega=\xi e^{+Q},
$$

where $\xi$ is a polynomial $N-2$ form. Then equation (3.13) becomes

$$
{ }^{*} v=P d \xi+2 d P \wedge \xi-P d Q \wedge \xi .
$$

We have found numerous examples of dissipative chaotic systems of this type, for which the real zeros of $\mathrm{P}$ do not form a closed manifold.

As an example with $N=3$, consider

$$
\begin{aligned}
P & =\left(1-x^{2}-z^{6}\right), \\
Q & =x^{4}+y^{4}+z^{2}+3 x y z, \\
\xi & =x y z d y+y^{2} d z .
\end{aligned}
$$

\footnotetext{
${ }^{5}$ The path integral measure in quantum field theories is often of this type. Note however that the domain of support may be restricted (for the ergodic measure) in a way which does not occur in quantum field theory.
} 
Planar projections of points generated by the resulting dynamical system (5.33) (observed at sufficiently long intervals) and by random sampling according to the probability distribution (5.31) appear consistent (see figure 5) upon restricting the domain of support of the probability distribution to $y>0$ and $z>0$.
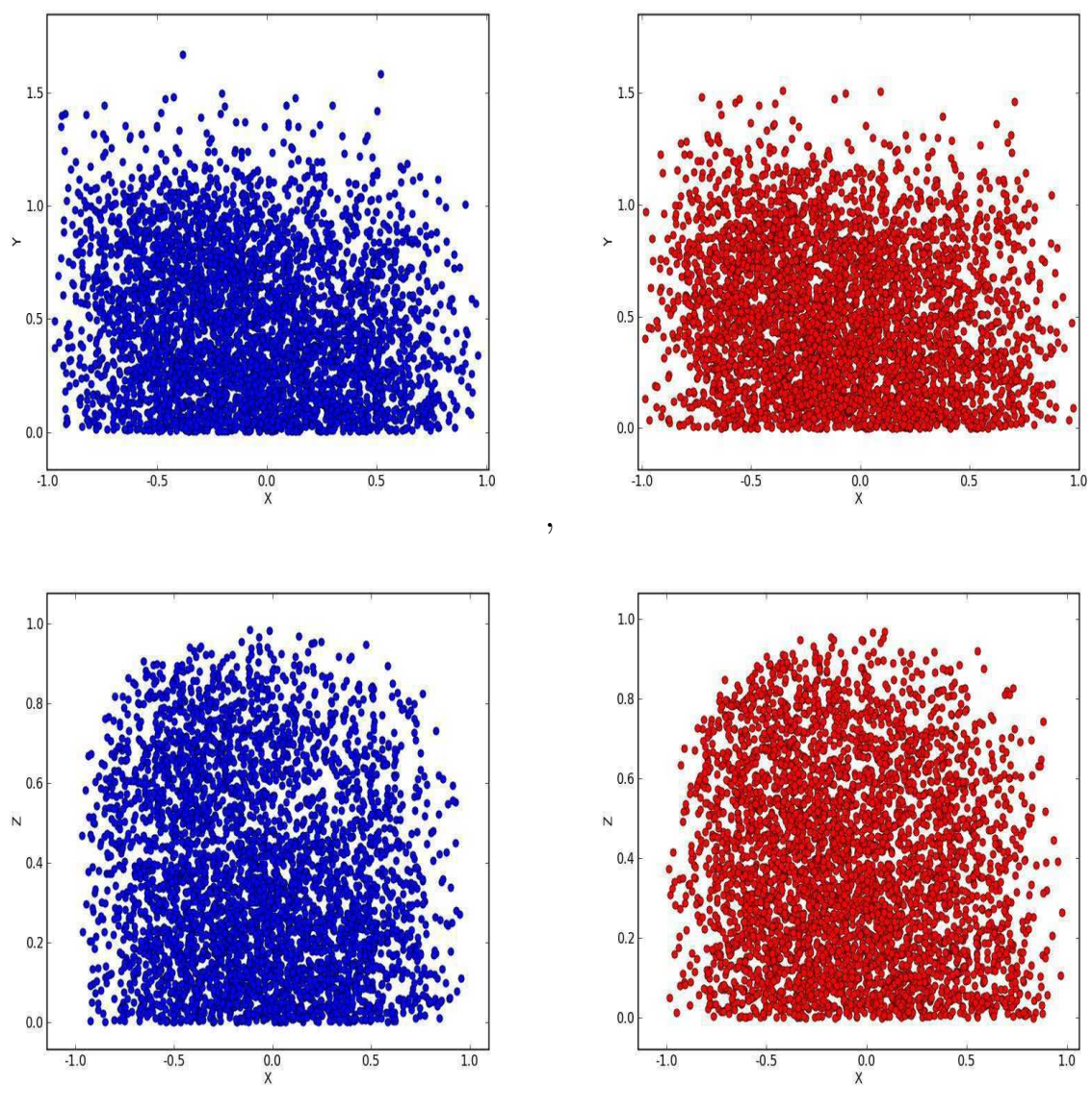

Figure 5. The XY and XZ projection of points (on the left) generated by the dynamical system defined by (5.34), observed at time intervals $\Delta t=1$ starting from the initial condition $(x, y, z)=$ $(0.1,1.1,0.4)$, and points (on the right) generated by random sampling with the probability distribution (5.35).

Comparing connected moment:6 associated with the probability distribution

$$
\begin{aligned}
& \rho=\left(1-x^{2}-z^{6}\right) e^{-\left(x^{4}+y^{4}+z^{2}+3 x y z\right)} \text { for } x^{2}+z^{6} \leq 1, y>0, z>0 \\
& \rho=0 \text { elsewhere. }
\end{aligned}
$$

with moments generated by the chaotic trajectory suggests that the two agree. (see table 3 ).

\footnotetext{
${ }^{6}<x y>_{c} \equiv<x y>-<x><y>$

$<x y z>_{c} \equiv<x y z>-<x y>_{c}<z>-<x z>_{c}<y>-<y z>_{c}<x>-<x><y><z>$, etc...
} 


\begin{tabular}{|c|c|c|}
\hline Moments & Dynamics & Monte Carlo \\
\hline$\langle<x>$ & -0.08497 & -0.09152 \\
\hline$\langle y>$ & 0.4898 & 0.5046 \\
\hline$\langle z>$ & 0.3712 & 0.3813 \\
\hline$<x^{2}>_{c}$ & 0.1697 & 0.1685 \\
\hline$\left.<y^{2}\right\rangle_{c}$ & 0.1052 & 0.1028 \\
\hline$<z^{2}>_{c}$ & 0.06145 & 0.05809 \\
\hline$<x y\rangle_{c}$ & -0.01830 & -0.01887 \\
\hline$\langle x z\rangle_{c}$ & -0.01304 & -0.01221 \\
\hline$<y z\rangle_{c}$ & 0.001292 & 0.0033309 \\
\hline$<x^{3}>_{c} /\left(<x^{2}>_{c}\right)^{3 / 2}$ & 0.1953 & 0.1946 \\
\hline$<y^{3}>_{c} /\left(<y^{2}>_{c}\right)^{3 / 2}$ & 0.4361 & 0.4119 \\
\hline$<z^{3}>_{c} /\left(<z^{2}>_{c}\right)^{3 / 2}$ & 0.3828 & 0.3027 \\
\hline$\left\langle x y z>_{c} / \sqrt{<x^{2}>_{c}<y^{2}>_{c}<z^{2}>_{c}}\right.$ & -0.07400 & -0.07370 \\
\hline$<x^{4}>_{c} /<x^{2}>_{c}^{2}$ & -0.7019 & -0.7433 \\
\hline & $\cdot$ & \\
\hline
\end{tabular}

Table 3. Entries in the column labeled 'dynamics' were obtained by mumerical simulation of the dynamical system over a time duration 40000 , sampling at intervals $\Delta t=0.1$, starting from the initial conditions $(x, y, z)=(0.1,1.1,0.4)$. The column labeled 'Monte Carlo' was obtained by a hit and miss Monte Carlo calculation using the probability distribution (5.35) with 400000 accepted data points.

Note that the distribution (5.35) seems to accurately reproduce the connected moment $<x y z>_{c}$ of the chaotic trajectory, which would not be expected if (5.35) only described the statistics of the trajectory upon planar projection. This could mean that the dimension of the chaotic invariant set, which we have not yet made an effort to calculate, is very nearly three. If not, then perhaps (5.35) has an interpretation as the Fourier transform of the the Hopf functional

$$
\Psi(\vec{j}) \equiv \lim _{T \rightarrow \infty} \frac{1}{T} \int_{0}^{T} d t \exp (i \vec{j} \cdot \vec{x}(t))
$$

and therefore determines all polynomial moments $\left\langle x^{n} y^{m} z^{l}\right\rangle$, although it must fail to give some other expectation values 7 . This assumes that the Hopf functional is sufficiently well behaved that its Fourier transform has support in $N$ dimensions.

This particular chaotic system behaves both as a repeller and attractor, depending on the initial conditions. trajectories outside with $x^{2}+z^{6}>1$ can never enter the chaotic invariant set. On the other hand initial conditions with $x^{2}+z^{6}<1$ and large $z$ are rapidly drawn into the region depicted in figure 5 .

Although we will not give explicit examples here, the inverse approach can also be applied to distributions with significantly more complicated analytic structure. For instance, one can

\footnotetext{
${ }^{7}$ One can always describe a distribution with support over a d-dimensional subspace of an $N$ dimensional space in terms of a distribution $\rho$ with support in $N$ dimensions together with a projection. However $\rho$ will only accurately determine expectation values of a subset of functions $f(\vec{x})$ on the $N$ dimensional phase space.
} 
take a distribution of the form

$$
\rho=\frac{P_{1}}{P_{2}} e^{\left(-P_{3} / P_{4}\right)}
$$

where $P_{i}$ are polynomials, together with the $N-2$ form

$$
\mathcal{A}=P_{1}^{2} P_{4}^{2} e^{\left(-P_{3} / P_{4}\right)} \xi
$$

where $\xi$ is a polynomial $N-2$ form. Then the velocity field $v={ }^{*} d \mathcal{A} / \rho$ will be polynomial. One must then verify that the distribution (or projected distribution), with some restricted domain of support, is ergodic. Although (5.37) may have real poles and essential singularities, these present no problem if they are integrable or lie outside the domain of support of the ergodic distribution.

\section{A remark about non-uniqueness of $\rho$ and conserved quantities}

Suppose that there is a variation $\rho \rightarrow \rho+\delta \rho, \mathcal{A} \rightarrow \mathcal{A}+\delta \mathcal{A}$ under which the dynamical system $v={ }^{*} d \mathcal{A} / \rho$ is invariant. This requires that

$$
-\frac{\delta \rho}{\rho} d \mathcal{A}+d(\delta \mathcal{A})=0
$$

Taking the exterior derivative of this expression gives

$$
d\left(\frac{\delta \rho}{\rho}\right) \wedge d \mathcal{A}=0
$$

or equivalently

$$
d\left(\frac{\delta \rho}{\rho}\right) \wedge^{*} v=0
$$

The last equation implies that $\delta \rho / \rho$ is a conserved quantity.

Note that in two dimensions, $\mathcal{A}$ is a zero-form and (6.39) always has a solution:

$$
\begin{aligned}
\frac{\delta \rho}{\rho} & =\epsilon \mathcal{A}, \\
\delta \mathcal{A} & =\frac{1}{2} \epsilon \mathcal{A}^{2},
\end{aligned}
$$

where $\epsilon$ is an infinitesimal constant. Since $\int \delta \rho$ must vanish, $\mathcal{A}$ must have zero mean with respect to $\rho$. This can always be arranged by taking $\mathcal{A} \rightarrow \mathcal{A}-\langle\mathcal{A}\rangle$, which does not change the dynamical system. The existence of this solution is a reflection of the fact that there can be no chaos with just two degrees of freedom. Any non-trivial invariant distribution $\rho$ is a distribution over closed orbits on which $\mathcal{A}$ is constant, but does not describe the statistics of a system with a given initial condition.

In some instances (in any dimension), the invariant probability distribution $\rho$ used to reverse engineer a dynamical system will not characterize individual trajectories because of the existence 
of conserved quantities. This does not necessarily exclude chaos, unless the number of conserved quantities is too large. The distribution $\rho$ can then be interpreted as a product of distribution over conserved quantities (which can be arbitrary) and a distribution which is dependent on the dynamics.

As a word of caution, not all conserved quantities associated with non-uniqueness of the invariant distribution $\rho$ impose strong constraints on trajectories. Suppose for example that a dynamical system admits two invariant distributions $\rho_{1}$ and $\rho_{2}$ with different non-overlapping but contiguous domains of support. Then there is a class of non-ergodic distributions of the form $x \rho_{1}+(1-x) \rho_{2}$ where $0 \leq x \leq 1$. The associated conserved quantity is $\left(\rho_{1}-\rho_{2}\right) /\left(\rho_{1}+\rho_{2}\right)$, which is 1 in the region of support of $\rho_{1}$ and -1 in the region of support of $\rho_{2}$. The only constraint this conserved quantity places on trajectories is that they can not go from the interior of region 1 to the interior of region 2 , or vice versa.

\section{Exponential distributions with Gaussian random forcing and Chaotic/Stochastic quantization}

Consider the static Fokker-Planck equation for a dynamical system in the presence of a Gaussian random force;

$$
d^{*}(\rho v-\Gamma d \rho)=0 .
$$

One can always (locally) write

$$
{ }^{*}(\rho v-\Gamma d \rho)=d \mathcal{A},
$$

for some $N-2$ form $\mathcal{A}$. Equivalently;

$$
v=\frac{1}{\rho}\left({ }^{*} d \mathcal{A}+\Gamma d \rho\right) .
$$

Assuming $\rho=\exp (-Q)$ and $\mathcal{A}=\xi \exp (-Q)$, where $Q$ is a polynomial and $\xi$ is a polynomial $N-2$ form, (7.45) becomes

$$
v={ }^{*}(d \xi-d Q \wedge \xi)-\Gamma d Q .
$$

The case $\xi=0$ is well known to quantum field theorists and arises in the context of stochastic quantization of a field theory with action $Q$ [10]. The dynamical system in this case has fixed points where $d Q=0$ with quantum fluctuations arising from Gaussian noise. Allowing nontrivial choices for $\xi$ gives a infinite class of possible quantizations. In the deterministic case, $\Gamma=0$, one must take care that $\xi$ is chosen such that the the dynamical system is chaotic.

As a highly speculative remark intended for quantum field theorists, we suggest that there may be an advantage to considering deterministic chaotic quantizations for which the distribution $\exp (-Q)$ is not ergodic. It has been noted earlier, in section 3 , that the initial distribution $\rho$ used in the inverse method may have symmetries which are not reflected by the dynamics. Phenomena in quantum field theory such as spontaneous symmetry breaking might be naturally encoded in the two-form ${ }^{*} \xi$, whereas stochastic quantization (with $\xi=0$ ) does not naturally give rise to symmetry breaking (see [8, 9] for potentially related discussions). If $\exp (-Q)$ is not ergodic, one must make sure that the Schwinger action principle equations which define quantum field theories are still satisfied. 


\section{Conclusions}

We have demonstrated an inverse method to construct chaotic dynamical systems starting from analytic expressions for an invariant probability distribution and a two-form. In principle, the inverse method can be used to generate an unlimited number of chaotic systems together with very well motivated conjectures for their exact statistics.

At present, the examples of dynamical systems we have considered are not physically motivated and in some cases are very complex, having a large number of terms in the components of the velocity field. The next challenge is to attempt to reverse engineer systems close to ones of physical interest. It would ultimately be very interesting to apply the inverse method to physical systems with a very large number of degrees of freedom, such as fluids. The most difficult part of reverse engineering a physical system seems to lie in choosing the two-form, which has no direct physical interpretation. Choosing a probability distribution is easier; one could, for instance, choose a distribution resembling that of a fluid with some mean shear.

Thus far, we have studied systems with only three or four degrees of freedom. Equations of the form (3.13) can be evaluated very quickly by hand or by computer, depending on the system, even for very large numbers of degrees of freedom. Potential computational problems arise only when trying to determine the domain of support of the ergodic distribution. At present, we do not have a way to do this besides direct numerical simulation of the dynamical system, which requires more computer time. However, allowing for this computer time, one should also be able to make very well motivated conjectures about the exact statistics of chaotic dynamical systems with a very large number of degrees of freedom.

Given an exact invariant distribution $\rho$, there are novel ways to compute time-series power spectra which will be discussed elsewhere. In particular, one can compute the power spectra by Monte-Carlo methods which are readily parallelizable. Moreover, it should also be possible to give analytic expressions for the asymptotic behavior of the power spectrum at high frequency.

While we have focused on developing an inverse method for dynamical systems on $\mathbb{R}^{N}$ with polynomial velocity fields, it may also be possible to consider more complicated phase space topologies such as $\mathbb{S}^{N} \otimes \mathbb{R}^{N}$, which are relevant to classical spin systems. It might also be interesting to reverse engineer dynamical systems on the $\mathrm{N}$-simplex, which arises in the context of evolutionary biology.

We wish to emphasize that even un-physical chaotic systems and their exact statistics, constructed by the inverse method, have an important use. Such systems can provide a testing ground for theoretical ideas. Moreover they can serve as benchmarks upon which various approximation methods, such as cumulant expansions and closure schemes for equations satisfied by mean quantities, can be tested.

\section{Acknowledgements}

I wish to thank Tibor Antal, Richard Brower, Stephen Libby, Brad Marston and Hisashi Ohtsuki for enlightening discussions. I would also like to thank the MIT center for theoretical physics for hospitality during completion of this work. 


\section{References}

[1] E. Hopf, Statistical hydromechanics and functional calculus, 1952 J. Ratl. Mech. Anal 187.

[2] U. Frisch, 1995 Turbulence, The Legacy of A. N. Kolmogorov (Cambridge: Cambridge University Press)

[3] J. B. Marston and O. Ma, Exact equal time statistics of Orszag-McLaughlin dynamics investigated using the Hopf Characteristic functional approach, J.Stat.Mech. (2005) P10007.

[4] H. Risken, 1996 The Fokker-Planck Equation: Methods of Solution and Applications 2nd edn (Berlin: Springer)

[5] E. A. Novikov, Functionals and the random-force method in turbulence, 1964 Sov. Phys. JETP 201290.

[6] S. A. Orszag, Lectures on the Statistical Theory of Turbulence, 1977 Fluid Dynamics, Les Houches 1973, ed R. Bailan and J. L. Peube ) (New York: Gordon and Breach)

[7] S. A. Orszag and J. B. McLaughlin, Evidence that random behavior is generic for nonlinear differential equations, 198 Physica D 168

[8] C. Pehlevan and G. Guralnik, Complex Langevin Equations and Schwinger-Dyson Equations, arXiv:0710.3756 [hep-th].

[9] G. Guralnik and Z. Guralnik, Complexified Path Integrals and the Phases of Quantum Field Theory, arXiv:0710.1256 [hep-th].

[10] G. Parisi and Y. Wu, Perturbation Theory Without Gauge Fixing Sci.Sin.24 (1981) 483. 